\title{
A rare case of subcutaneous zygomycosis on the neck of an immunocompetent patient
}

\author{
Maria Verónica Avendaño ${ }^{1}$, Scarlett Andreina Contreras ${ }^{2}$, Chetan Yuoraj Dhoble ${ }^{3}$, Alvaro Rafael \\ Camacho ${ }^{4}$, Mariangeles Medina ${ }^{4}$, Kana Riza Amari ${ }^{5}$ \\ 1. Los Andes University, Mérida-Venezuela, Venezuela. 2. Department of Internal Medicine, Faculty of Medicine in Los \\ Andes University, I Iahula Mérida, Venezuela. 3. N.K.P.Salve Institution of Medical Sciences and Research Center, India. 4. \\ Universidad de carabobo facultad de ciencias de la salud, Valencia, Venezuela. 5. Faculitad de Ciencias de la Salud, \\ Universidad de Carabobo, Venezuela
}

Correspondence: Chetan Yuoraj Dhoble. Address: N.K.P.Salve Institution of Medical Sciences and Research Center, India. Email: chetandhoble@gmail.com

Received: June 2, 2015 Accepted: July 21, $2015 \quad$ Online Published: July 24, 2015

DOI : 10.5430/crim.v2n3p57 URL: http://dx.doi.org/10.5430/crim.v2n3p57

\begin{abstract}
Zygomycosis is a sporadic entity affecting subcutaneous tissue characterized by painless gradually growing nodules that often appear on the trunk, extremities, and occasionally in other regions. It is included among the diseases that cause the chronic granulomatous reaction of the tissues. It predominantly affects immunocompromised patients by invading the brain, lungs, and gastrointestinal tract, but it has also been reported in immunocompetent patients with manifestations primarily in the cutaneous and subcutaneous tissues. The relatively low incidence of this disease limits clinical suspicion, often leading to a delay in diagnosis. Here we present the case of an immunocompetent 18-year-old Venezuelan male, with a rapidly growing neck mass that was earlier misdiagnosed as Tuberculosis. The diagnosis was made by tissue biopsy that revealed the presence of thin-walled hyphae, often chambered or fragmented, surrounded by an eosinophilic sheath (Splendore-Hoeppli phenomenon) which is a significant feature of this mycosis. Once diagnosed, the patient responded well to medical management with potassium iodide. Zygomycosis causes a wide spectrum of pathologies in immunocompromised patients. However, there should be a high clinical suspicion to establish its diagnosis, which is particularly important in atypical immunocompetent patients.
\end{abstract}

\section{Keywords}

Zygomycosis, Subcutaneous tissue, Nodules, Granulomatous reaction, Immunocompetent

\section{I ntroduction}

The incidence of subcutaneous mycosis has increased in recent years due to the growing prevalence of diseases affecting the immune system such as HIV and cancer ${ }^{[1,2]}$. Among these, the zygomycosis represents a rare infection caused by fungi belonging to the zygomycetes class that are divided into two orders: Mucorales and Entomophthorales. The families of Mucorales causes an infection called Mucormycosis ${ }^{[3]}$. The Entomophthorales order has three major pathogenic species: Basidiobolus ranarum, Conidiobolus coronatus, and Conidiobolus incongruus. Basidiobolus ranarum causes an infection called subcutaneous zygomycosis that often occurs in the trunk, extremities and occasionally in other regions ${ }^{[4]}$. The actual incidence of this disease around the world is unknown because it is rarely diagnosed or reported. Most of the 
reported cases have occurred in Africa and India with a few reports in Latin America and the United States ${ }^{[4]}$. In Venezuela, there have been six biopsy-confirmed cases ${ }^{[5]}$. $90 \%$ of these cases have been described in individuals under 20 years of age with a male predominance of about 3:2; almost all reported cases have occurred in immunocompromised individuals ${ }^{[4-6]}$. The exact mechanism of transmission is unknown. It has been postulated that zygomycetes such as $B$. ranarum, grows on vegetation and organic waste consumed by insects and other arthropods. These in turn are eaten by frogs, lizards and other animals who serve as vectors. They spread the fungus and infect humans either by inhalation of spores, direct trauma with contaminated objects or by contact with a previously injured epithelial barrier ${ }^{[3,5]}$. In any case, diagnosis of zygomycosis is especially difficult because few clinicians have both, enough experience to recognize it and to feel secure in the diagnosis and treatment of this disease ${ }^{[3]}$. We report the case of an 18-year-old Venezuelan male with a rapidly growing cervical tumor who presented with diagnostic difficulties.

\section{Case presentation}

An 18-year-old Venezuelan male with a history of a refractory neck mass was admitted in September of 2012 for treatment. The patient had first reported a right-sided neck mass 19 months prior. It showed progressive enlargement extending posteriorly and circumferentially around the neck. The mass was painless, erythematous, and occasionally produced a serous discharge. Oddly, the patient reported that the erythema over the mass would continually reappear and resolve spontaneously. The patient reported a limited range of motion of his neck. He had been hospitalized on several occasions without a definitive diagnosis. On one of those occasions, first line anti-tuberculosis drugs (isoniazid, rifampin, pyrazinamide, and ethambutol) were begun due to the high prevalence in the region. The patient reported having received this therapy during four months without any benefit. He reported no significant medical history, medications, or allergies. Family history was noncontributory. Of note, the patient lived in a rural area and had been exposed to cattle and pigs. Review of systems was negative.
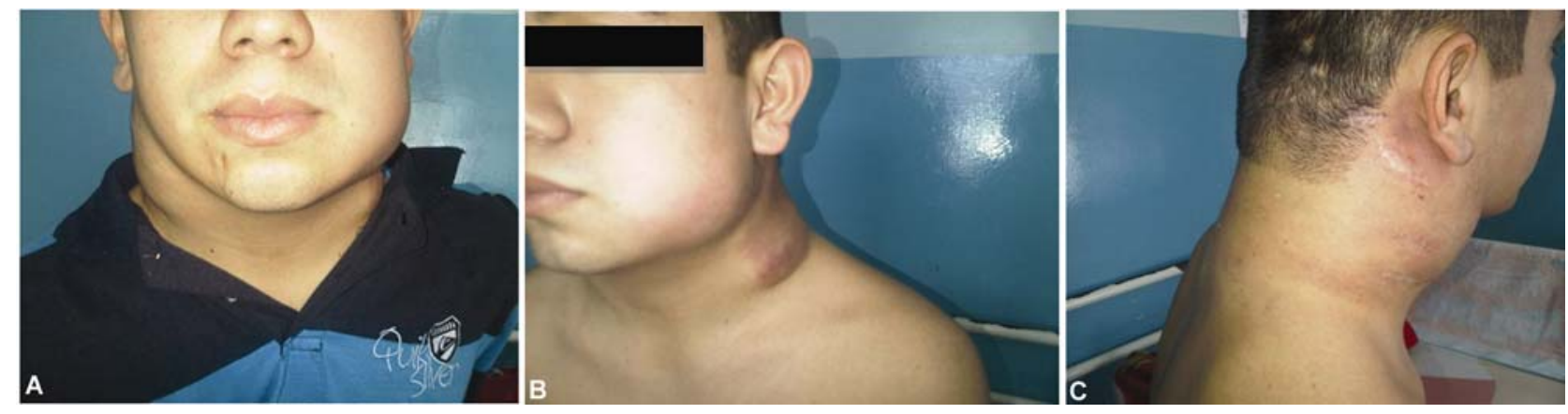

Figure 1. Tumors at varying times of the clinical course

On exam, vitals were within normal limits. He presented two masses: left mandibular mass and right cervical mass (see Figure 1). The left-sided mass was a nontender, immobile, well circumscribed $15 \mathrm{~cm} \times 15 \mathrm{~cm}$ mass with a fluctuant and erythematous center. The right-sided mass was a nontender, immobile, well circumscribed $10 \mathrm{~cm} \times 15 \mathrm{~cm}$ mass without erythema or fluctuation. The mass extended posteriorly but spared the midline. Neck rotation was limited bilaterally. No lymph nodes were palpated. The rest of the physical exam was benign.

CBC showed a leukocyte count of 18,000 with eosinophilia of 31\%. Hemoglobin was low at 12 with an elevated LDH of 424. Soft tissue ultrasound of the neck showed distortion and thickening of subcutaneous tissues bilaterally with areas of necrosis and cervical lymphadenopathy. CT scan of the head and neck demonstrated three distinct masses infiltrating the subcutaneous tissue with lateral cervical lymphadenopathy. The measured $8 \mathrm{~cm} \times 5 \mathrm{~cm}, 6 \mathrm{~cm} \times 7 \mathrm{~cm}$, and $5 \mathrm{~cm} \times 14 \mathrm{~cm}$ with irregular contours (see Figure 2). The cervical spine and trachea were unaffected. 
Lab tests were negative for Aspergillus, Candida, Histoplasma, Sporothrix, and Paracoccidioides. Tissue specimen examination for tuberculosis with Ziehl Neelson and PCR was negative. His PPD and X-ray chest were negative (see Figure 3). Extensive workup with cultures, antigen detection, antibody detection, and PCR were negative for fungi, viruses, and tuberculosis. Of note, HIV, Syphilis, Toxoplasma, Rubella, and EBV/CMV were not detected. Bone marrow aspirate (10/10/2012) showed myeloid Series 270 cells. 54\% (Reference: 49\%-65\%), erythroid series 123 cells, 24.5\% (R: 18\%-33\%), Lymphoid Series 107 cells, 21\% (R: 11\% to 23\%). Bone marrow biopsy (10/10/2012) revealed normal cellularity.

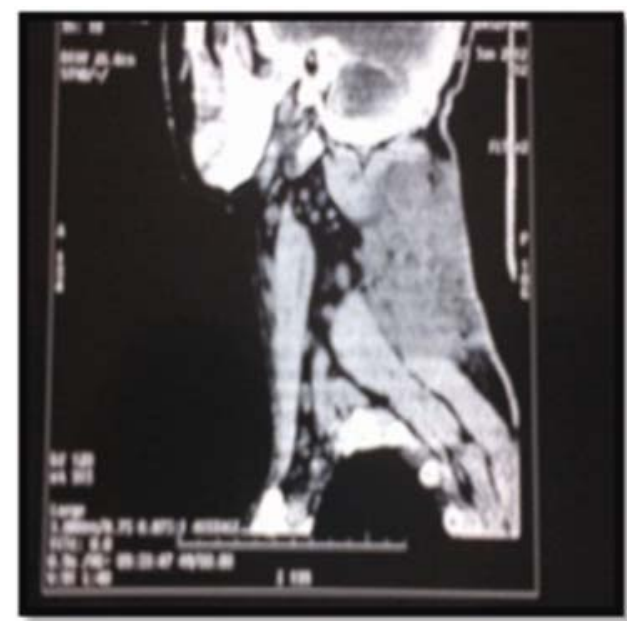

Figure 2. CT scan of the neck

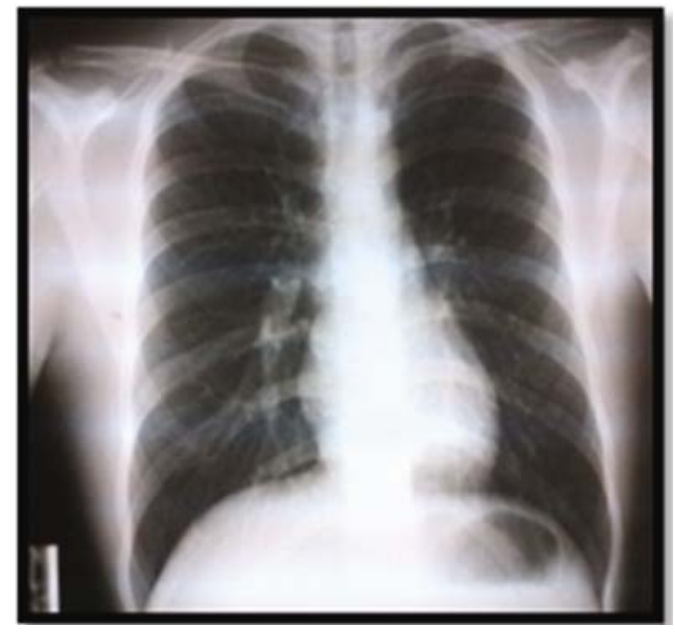

Figure 3. Chest X-ray

Per records, three previous biopsies had been performed showing Langhans multinucleated giant cells with areas of necrosis and acid-fast (AFB) negative, without evidence of fungi. Two biopsies of the mass at our institution showed similar findings. Consistent with the finding in biopsies and the previously unremarkable workup, the differential diagnosis included granulomatous pathologies, lymphoproliferative diseases, and other neoplastic pathologies. Despite the prevalence of tuberculosis (TB), AFB was negative in all samples. Additionally the patient reported having completed anti-tuberculosis therapy. Despite eosinophilia, a common finding in Hodgkins disease, biopsy of the mass and bone marrow did not show malignancy. Therefore, with eosinophilia and granulomatous reaction on biopsy, we started empirical antifungal treatment with itraconazole. The patient remained in stable condition until December 2012 when he began to experience difficulty opening his mouth. Stridor was observed during the exam and, for this reason, a steroid treatment was started improving his symptoms.

A sixth biopsy was performed and sent to an outside institution (Medical College of Wisconsin, USA, 12/14/2012). The biopsy showed subcutaneous chronic granulomatous inflammation and necrosis. Within the foci of necrosis and inside the giant cells, fungal elements were seen characterized by short, broad and irregular septate hyphae, usually showing the call Splendore-Hoeppli reaction (see Figure 4). The special stains for fungi highlight those elements (Grocott-Gomori methenamine-silver stain and Periodic acid Schiff). AFB was negative.

Although infection by some bacilli such as Nocardia and Mycobacterium tuberculosis can cause the Splendore-Hoeppli reaction, the negativity for acid alcohol resistance and the presence of fungal elements rule out those agents. Conversely, this reaction is associated with the presence of septate hyphae which is a pathognomonic feature of zygomycetes class ${ }^{[7]}$ and also excluded dimorphic fungi such as Sporothrix. Therefore, this study is conclusive for zygomycosis. 


\section{Discussion}

The first case of zygomycosis was published by Paultauf in $1885^{[8]}$, since then around 160 cases have been described in the literature, despite the difficulty that represents recognizing and reporting it ${ }^{[4]}$. The exposed areas of the extremities are the most commonly affected; although the head and neck are quite exposed, they are less frequently affected. The typical clinical presentation is a solitary, firm, subcutaneous nodule. It causes no pain and gradually grows radially outward, attached to the skin and deeper layers ${ }^{[4]}$. This presentation can mimic a soft tissue tumor ${ }^{[9]}$. The main route of transmission of this fungus is via inhalation of spores, or by a traumatic introduction of spores has been documented. In a considerable number of patients: via catheter insertion, intravenous drug use, in tattooing, use of adhesives over wounds, and insect bites. Less common is ingestion ${ }^{[3]}$. In this case, the long period between inoculation and presentation made it difficult to determine the specific source. Diagnosis of subcutaneous zygomycosis requires special collection, culturing and stain techniques (see Figure 5), which explain the fact that up to $50 \%$ of cases in some series are only diagnosed postmortem ${ }^{[3,4]}$. Isolation of zygomycetes in patient samples offers little clinical value since patients can be colonized by the fungus without infection, many of these due to treatment with steroids or broad spectrum antibiotics. Furthermore, the fungus can contaminate lab cultures ${ }^{[3]}$. Microscopically, the acute reaction shows eosinophils, lymphocytes, and plasma cells. Later, a chronic granulomatous reaction occurs. The typical histopathological feature of zygomycetes such as $B$. ranarum is the presence of thin-walled broad hyphae or hyphal fragments frequently phagocytized within giant cells ${ }^{[4]}$. Additionally, a common finding is the Splendore-Hoeppli phenomenon or asteroid bodies, consisting of intensely eosinophilic material, radiated in star-shaped around microorganisms ${ }^{[10]}$. Splendore-Hoeppli is a phenomenon that may be present in both, bacterial infections (botryomycosis, nocardiosis and actinomycosis) and other fungal infections (sporotrichosis, Pityrosporum folliculitis, candidiasis, aspergillosis and blastomycosis). It has also been described in some parasitic diseases ${ }^{[10]}$. Therefore, it is only a guide in the diagnostic process. All this agents have distinctive morphology and clinical features that in our case, allowed its exclusion ${ }^{[11]}$.

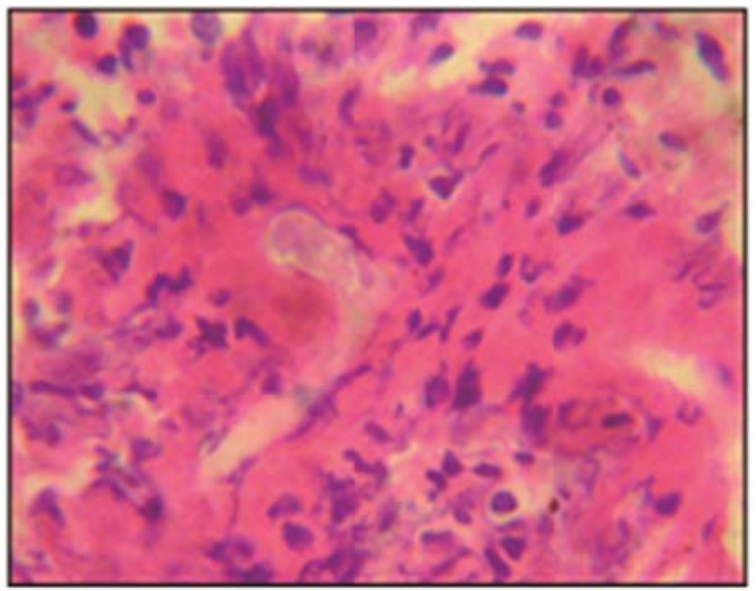

Figure 4. Splendore-Hoeppli phenomenon illustration

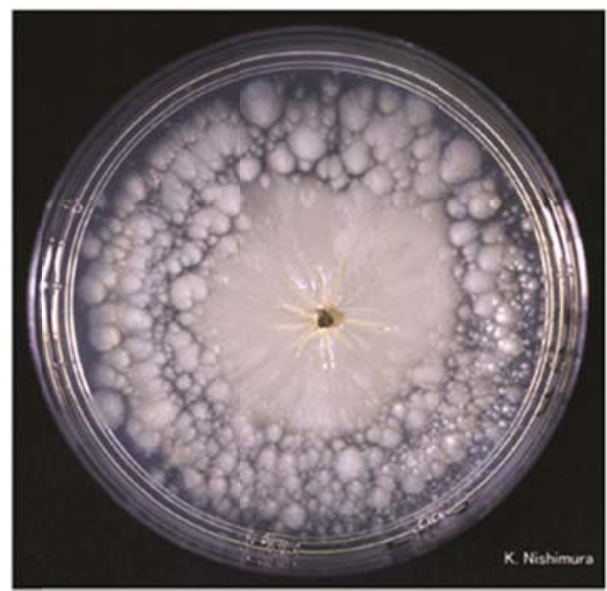

Figure 5. B. ranarum colony illustration

Humans have an effective and rapid response to zygomycetes. Therefore, deficiency of phagocytic cells is one of the main risk factors; either deficit in number (neutropenia) or decline in function (steroid use and ketoacidosis). Thus, zygomycotic infections are characteristic of immunosuppressed patients ${ }^{[6]}$. This particular factor could not be demonstrated in our patient. In immunocompetent patients, zygomycoses produce a pattern of predominantly skin and subcutaneous tissue infections. The dependence of the primary infection site on the immunocompetence of the host is illustrated in a review of 929 cases by Roden et al. ${ }^{[12]}$. Treatment can be both surgical and antifungal therapy. Amphotericin B, with a very low MIC, is the most potent antifungal available ${ }^{[6]}$ but is largely ineffective in the most advanced cases ${ }^{[13]}$. Other antifungals can be used for treating $B$. ranarum. In few studies, potassium iodide was used as the first alternative ${ }^{[4,14]}$, placing co-trimoxazole, miconazole, ketoconazole and itraconazole as a secondary treatment ${ }^{[5,15,16]}$. Voriconazole by contrast has a very high MIC and is clinically ineffective in invasive zygomycoses ${ }^{[3]}$. Our patient showed a poor response to 
itraconazole and intolerance to amphoteric B. It was decided to initiate treatment with posaconazole 200 mg every 8 hours as per the recommended guidelines ${ }^{[6]}$. Due to the high cost and low availability of the drug in the country, the decision was made to alter therapy by starting potassium iodide ( $30 \mathrm{mg} / \mathrm{kg} / \mathrm{day})$. The patient showed a marked clinical improvement in almost five months of subsequent treatment as shown in Figure 6.
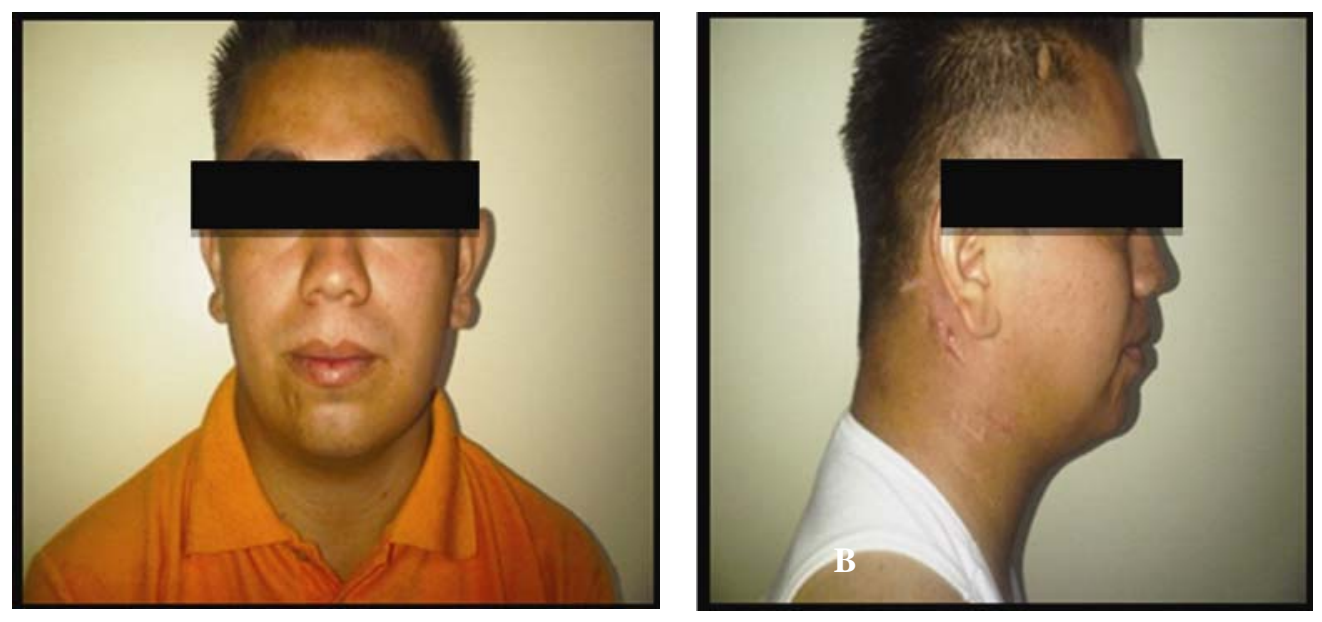

Figure 6. Patient after completing the treatment for zygomycosis in April 2013

Zygomycosis has been a recognized entity for many years, affecting more commonly the immunocompromised patients. However, there should be a high clinical suspicion to establish its diagnosis, especially in atypical immunocompetent patients.

\section{Acknowledgements}

Illustrations are courtesy of www.pf.chiba-u.ac.jp/eng/gallery/index.html.

\section{References}

[1] Manterola JM. Fungal disease. Med Clin (Barc). 2001; 117: 96-8.

[2] Dolande M, Reviákina V, Panizo M, et al. Immunological diagnosis of systemic fungal infections in patients with AIDS (1997-2001). Review of the Venezuelan Society of Microbiology. 2002; 22: 51-6.

[3] M Torres-Narbona JG, Munoz P, Bouza E. Zygomycetes and zygomycoses in the era of new antifungal therapies. Spanish Journal of Chemotherapy. 2007; 20: 375-86. PMid:18563211

[4] Gugnani HC. A review of zygomycosis due to Basidiobolus ranarum. Eur J Epidemiol. 1999; 15 (10): 923-9. PMid:10669127 http://dx.doi.org/10.1023/A:1007656818038

[5] Cermeño JJ, Cermeño JR, Mujica M. Zigomicosis en el Complejo Hospitalario Universitario “Ruiz y Páez”, Ciudad Bolívar, período 1984-2007. Rev. Soc. Venez. Microbiol. 2008; 28(1): 20-23.

[6] Arias GGJ. Zygomycosis. Infectio. 2010; 14: 181-92. http://dx.doi.org/10.1016/S0123-9392(10)70135-1

[7] Rippon JW. Medical Mycology-The Pathogenic Fungi and the Pathogenic Actinomycetes. Third Edition. Philadelphia: J. Basic Microbiol. 1990; 30:463.

[8] Paultauf A. Mycosis mucorina. Path Arch Anat. 1885; 102(3): 543-564. http://dx.doi.org/10.1007/BF01932420

[9] Thotan SP, Kumar V, Gupta A, et al. Subcutaneous Phycomycosis-Fungal Infection Mimicking a Soft Tissue Tumor: A Case Report and Review of Literature. Journal of tropical pediatrics. 2010; 56(1): 65-66. PMid:19520731 http://dx.doi.org/10.1093/tropej/fmp049

[10] Hussein MR. Mucocutaneous Splendore-Hoeppli phenomenon. J Cutan Pathol. 2008; 35(11): 979-88. PMid:18976399 http://dx.doi.org/10.1111/j.1600-0560.2008.01045.x 
[11] Pfaller MA, Diekema DJ. Unusual Fungal and Pseudofungal Infections of Humans. Journal of clinical microbiology. 2005; 43(4p): 1495-1504. PMid:15814958 http://dx.doi.org/10.1128/JCM.43.4.1495-1504.2005

[12] Roden MM, Zaoutis TE, Buchanan WL, et al. Epidemiology and outcome of zygomycosis: a review of 929 reported cases. Clinical Infectious Diseases. 2005; 41(5): 634-653. PMid:16080086 http://dx.doi.org/10.1086/432579

[13] Curutchet D. Zygomycosis. Argentina Journal of Dermatology. 2010; 91 (2).

[14] Anand M, Deshmukh SD, Pande DP, et al. Subcutaneous zygomycosis due to Basidiobolus ranarum: a case report from Maharastra, India. Journal of tropical medicine. 2010(2010).

[15] Sabatelli F, Patel R, Mann PA, et al. In vitro activities of posaconazole, fluconazole, itraconazole, voriconazole, and amphotericin B against a large collection of clinically important molds and yeasts. Antimicrobial Agents and Chemotherapy. 2006; 50(6): 2009-2015. PMid:16723559 http://dx.doi.org/10.1128/AAC.00163-06

[16] Torres-Narbona M, Guinea J, Martínez-Alarcón J, et al. In vitro activities of amphotericin B, caspofungin, itraconazole, posaconazole, and voriconazole against 45 clinical isolates of zygomycetes: comparison of CLSI M38-A, Sensititre YeastOne, and the Etest. Antimicrobial agents and chemotherapy. 2007; 51(3): 1126-1129. PMid:17194821

http://dx.doi.org/10.1128/AAC.01539-06 